\title{
Comparability and repeatability of different methods of corneal astigmatism assessment
}

This article was published in the following Dove Press journal:

Clinical Ophthalmology

\author{
Tiago B Ferreira \\ Filomena J Ribeiro \\ Department of Ophthalmology, \\ Hospital da Luz, Lisbon, Portugal
}

Correspondence: Tiago B Ferreira Department of Ophthalmology, Hospital da Luz, Avenida Lusiada 100, Lisboa 1500-650, Portugal

Tel +35I 217 I0 4400

$\mathrm{Fax}+351217104409$

Email tiagoferreira@netcabo.pt
Purpose: To assess the comparability and repeatability of keratometric and astigmatism values measured by four techniques: Orbscan IIz ${ }^{\circledR}$ (Bausch and Lomb), Lenstar LS 900 ${ }^{\circledR}$ (Haag-Streit), Cassini $^{\circledR}$ (i-Optics), and Total Cassini (anterior + posterior surface), in healthy volunteers.

Patients and methods: Fifteen healthy volunteers (30 eyes) were assessed by the four techniques. In each eye, three consecutive measures were performed by the same operator. Keratometric and astigmatism values were recorded. The intraclass correlation coefficient (ICC) was used to assess comparability and repeatability. Agreement between measurement techniques was evaluated with Bland-Altman plots.

Results: Comparability was high between all measurement techniques for minimum keratometry (K1), maximum keratometry (K2), astigmatism magnitude, and astigmatism axis, with ICC $>0.900$, except for astigmatism magnitude measured by Cassini compared to Lenstar ( $I C C=0.798$ ) and Orbscan compared to Lenstar ( $I C C=0.810)$. However, there were some differences in the median values of $\mathrm{K} 1$ and $\mathrm{K} 2$ between measurement techniques, and the Bland-Altman plots showed a wide data spread for all variables, except for astigmatism magnitude measured by Cassini and Total Cassini. For J0 and J45, comparability was only high for J0 between Cassini and Orbscan. Repeatability was also high for all measurement techniques except for K2 (ICC =0.814) and J45 (ICC =0.621) measured by Cassini.

Conclusion: All measurement techniques showed high comparability regarding K1, K2, and astigmatism axis. Although posterior corneal surface is known to influence these measurements, comparability was high between Cassini and Total Cassini regarding astigmatism magnitude and axis. However, the wide data spread suggests that none of these devices should be used interchangeably.

Keywords: astigmatism, keratometry, topography, repeatability, comparability

\section{Introduction}

Accurate measurements of corneal power and astigmatism are crucial to attain optimal results in cataract surgery. ${ }^{1,2}$ Given the variety of currently available devices and the lack of a recognized gold standard, it is important to assess their comparability and repeatability, the former to evaluate the potential exchangeability of measurements obtained by different techniques and the latter to help establish which technique or instrument performs better. ${ }^{1}$ Although several studies have previously investigated the comparability and repeatability of different available devices regarding corneal power and astigmatism, ${ }^{3-6}$ namely the Cassini ${ }^{\circledR}$ (i-Optics) device used in this study, ${ }^{1,2,7,8}$ no study has compared the corneal power and astigmatism measured using Orbscan IIz ${ }^{\circledR}$ (Bausch and Lomb), Lenstar LS $900^{\circledR}$ (Haag-Streit), and Cassini and Total Cassini (the sum of the anterior and posterior corneal surfaces) in the same eyes and by the same operator. 
Cassini (i-Optics) is a new technology specifically developed to assess eyes before cataract surgery. ${ }^{9}$ This technology has been shown to have a very high specificity in estimating corneal keratometry even in post-LASIK, keratoconic, and cross-linked corneas, ${ }^{10}$ and also a high precision in reporting corneal astigmatism. ${ }^{11}$

The goal of this work was to assess the comparability and repeatability of keratometric and astigmatism values measured by four techniques: Orbscan IIz, Lenstar LS 900, Cassini, and Total Cassini (anterior + posterior surface), in healthy volunteers.

\section{Patients and methods}

\section{Setting and population sample}

This was an institutional cross-sectional study that included 15 healthy volunteers (30 eyes). All eyes were assessed by the four techniques. Three consecutive measures (10 minutes apart) were performed in each eye by the same operator, using the four techniques. Keratometric and astigmatism values were recorded. Inclusion criteria were healthy individuals aged $18-50$ years with a corrected visual acuity of $0.00 \log$ MAR or better. Exclusion criteria were a history of ocular pathology, trauma, contact lens wear, systemic or local medications, and ocular surgery. In addition, patients with anterior segment pathologies such as dry eye, Meibomian gland disease, corneal disease, or abnormal topographies were excluded from this study. All participants were in the proper head positioning, and targets were positioned as instructed by the manufacturer of each device. The sequence of the measurements with the three devices was randomly chosen. The study protocol was approved by Hospital da Luz Institutional Review Board. All participants provided written informed consent.

\section{Automated topographers}

Topography data were obtained with Orbscan IIz (Bausch and Lomb), Lenstar LS 900 (Haag-Streit), and Cassini (i-Optics).

Orbscan IIz is a Placido-based multidimensional system that combines slit-scan and Placido ring technology, providing a complete analysis of the corneal surface, evaluating all corneal curvatures. Keratometry from the $3.0 \mathrm{~mm}$ zone was used to maximize comparability between devices. The Lenstar LS 900 uses 32 measuring points arranged in two concentric rings (outer $2.3 \mathrm{~mm}$, inner $1.65 \mathrm{~mm}$ ) of 16 measuring points each. ${ }^{12}$ Cassini is a topographer that uses multicolor point-topoint (up to 700) ray tracing, combined with second Purkinje Imaging Technology. ${ }^{13}$ A detailed description of each topographer has been previously described. ${ }^{9}$ Keratometry is measured in the $3.0 \mathrm{~mm}$ zone.

Calibration of all topographers was performed according to the manufacturer's instructions.

\section{Measurements}

Assessed parameters were minimum (K1), maximum (K2), mean keratometry $(\mathrm{Km})$, astigmatism magnitude (D), and axis $\left(^{\circ}\right)$. For the Cassini, these values were recorded for the anterior corneal surface and for total corneal astigmatism (anterior + posterior surface). Vectors J0 and J45 were also assessed for the cardinal $\left(0^{\circ}-180^{\circ}\right)$ and oblique $\left(45^{\circ}-135^{\circ}\right)$ meridians and were calculated using the formulas $\mathrm{J} 0=\mathrm{D} \times \cos (2 \pi \times$ axis $/ 180)$ and $\mathrm{J} 45=\mathrm{D} \times \sin (2 \pi \times$ axis $/ 180)$, according to Thibos and Horner. ${ }^{14}$

Power vectors were conceived as a way of transforming conventional refractive error, or keratometric data, into mutually independent, orthogonal components, better suited to statistical analysis. Vector analysis permits a complete description of astigmatism characteristics ${ }^{15}$ and allows the comparison of both orientation and power.

\section{Statistical analysis}

After Shapiro-Wilk tests of all variables, the Wilcoxon test was used to compare measurements performed on the different pairs of devices. The intraclass correlation coefficient (ICC) was used to assess comparability and repeatability. The ICC expresses the consistency of repeated measurements, ranging from 0 to 1 . An ICC $<0.75$ indicates poor repeatability, from 0.75 to 0.89 moderate repeatability, and $>0.90$ high repeatability. ${ }^{1}$

When comparing axis, $180^{\circ}$ was added to or subtracted from the measured axis so that measurement differences between methods were never $>90^{\circ} .8,9,11$ Analysis of agreement between each pair of devices was performed using Bland-Altman plots. ${ }^{9,16}$ The $95 \%$ limits of agreement (LoAs) represent the limits of the range for the $95 \%$ of differences between each pair of devices. According to the Bonferroni correction, tests were considered significant at $P<0.008$ significance level (two-tailed). Data were processed using IBM SPSS 21 software (Armonk, NY, USA).

\section{Results}

\section{Comparability of keratometry readings}

Univariate analysis comparing $\mathrm{K} 1$ and $\mathrm{K} 2$, as assessed by Total Cassini, Cassini, Orbscan, and Lenstar, showed that there were differences in the median values of $\mathrm{K} 1$ and $\mathrm{K} 2$ between Lenstar and Orbscan (Table 1). The ICC used 
Table I Descriptive statistics

\begin{tabular}{lllll}
\hline Parameter & Total Cassini & Cassini ${ }^{\circledR}$ & Orbscan IIz $^{\circledR}$ & Lenstar LS 900 $^{\circledR}$ \\
\hline KI (D) & & $42.77(36.93-46.06)$ & $42.90^{*}(36.90-45.70)$ & $42.82^{*}(36.86-45.76)$ \\
K2 (D) & & $44.31(41.24-47.06)$ & $44.17^{*}(40.87-46.43)$ & $44.06^{*}(41.32-46.63)$ \\
Astigmatism (D) & $0.88(0.44-2.04)$ & $0.89(0.25-5.80)$ & $0.80(0.27-2.17)$ & $0.87(0.34-3.04)$ \\
Axis $\left(^{\circ}\right)$ & $89.33(I 1.33-147.50)$ & $91.92(10.67-167.00)$ & $93.00(16.00-167.00)$ & $94.33(I 2.67-163.00)$ \\
J0 (D) & $0.23^{*}(-0.92$ to 0.72$)$ & $0.30^{*}(-0.86$ to 2.87$)$ & $0.33(-0.78$ to 2.82$)$ & $0.32(-0.82$ to I.48) \\
J45 (D) & $-0.01(-0.76$ to 0.36$)$ & $0.03^{*}(-0.79$ to 0.56$)$ & $0.04(-0.54$ to 1.63$)$ & $0.05^{*}(-0.45$ to 1.34$)$ \\
\hline
\end{tabular}

Notes: All values are presented as median (minimum-maximum). Orbscan showed higher values of $\mathrm{KI}$ and $\mathrm{K} 2$ compared to Lenstar. Cassini showed a higher value of J0 compared to Total Cassini and a lower value of $\mathrm{J} 45$ compared to Lenstar. $* P<0.005$.

Abbreviations: $\mathrm{K} 1$, minimum keratometry; $\mathrm{K} 2$, maximum keratometry.

to assess comparability showed that comparability was high between all measurement techniques for $\mathrm{K} 1$ and $\mathrm{K} 2$. Agreement between assessment methods for $\mathrm{Km}$ is further illustrated in the Bland-Altman plots (Figure 1). The Cassini vs Lenstar agreement regarding $\mathrm{Km}$ showed the closest to 0 mean difference $(-0.030)$ but the highest range of LoA (2.397). The Orbscan vs Lenstar agreement showed the highest mean difference $(-0.143)$ and the lowest range of LoA (0.301).

\section{Comparability of astigmatism evaluation}

Univariate analysis comparing astigmatism magnitude, astigmatism axis, J0, and J45, as assessed by Total Cassini, Cassini, Orbscan and Lenstar, showed that there were differences in the median values of $\mathrm{J} 0$ between Total Cassini and Cassini, and of J45 between Cassini and Lenstar (Table 1). The ICC used to assess comparability was high between all measurement techniques for astigmatism magnitude and astigmatism axis, with ICC $>0.900$, except for astigmatism magnitude measured by Cassini compared to Lenstar (ICC $=0.798)$ and Orbscan compared to Lenstar ( ICC $=0.810$ ). For J0 and J45, comparability was only high for J0 between Cassini and Orbscan (ICC $=0.989$ ), with all other comparisons showing ICC $<0.900$. Although all comparisons showed a $P<0.001$, J45 between Cassini and Orbscan and between Orbscan and Lenstar were notably low (ICC $=0.522$ and ICC $=0.690$, respectively). When comparing Cassini and Total Cassini regarding both astigmatism magnitude and axis, comparability was high $(\mathrm{ICC}=0.941$ and $\mathrm{ICC}=0.983$, respectively, $P<0.001$ ).

Agreement between assessment methods for astigmatism magnitude and astigmatism axis is further illustrated in the Bland-Altman plots (Figure 1). The best agreement for astigmatism magnitude was between Total Cassini and Cassini, having the closest to 0 mean difference $(0.014)$ and the lowest range of LoA (0.862). The agreement between Cassini and Lenstar showed the highest mean difference (0.201), and the Orbscan vs Lenstar the highest range of LoA (2.923). Regarding astigmatism axis, all comparisons showed a wide data spread, with the agreement between Total Cassini and Lenstar showing the closest to 0 mean difference (0.802), and the Total Cassini vs Cassini the lowest range of LoA (34.385). The agreement between Total Cassini and Cassini showed the highest mean difference (2.928), and the Total Cassini vs Orbscan the highest range of LoA (63.564).

\section{Repeatability}

The ICC used to assess repeatability showed that this was high for all measurement techniques and assessed parameters, with ICC $>0.900$, except for $\mathrm{K} 2$ and $\mathrm{J} 45$ measured by Cassini (ICC $=0.814$ and ICC $=0.621$, respectively) (Table 2).

\section{Discussion}

Given the importance of evaluating the potential exchangeability of corneal power and astigmatism measurements, ${ }^{1}$ we assessed the comparability and repeatability of keratometric and astigmatism values measured by four techniques - Orbscan IIz, Lenstar LS 900, Cassini, and Total Cassini (anterior + posterior surface) - in 30 eyes of 15 healthy volunteers.

\section{Comparability}

The results reported in the literature concerning comparability between devices are not consensual. Ventura et al found no differences in mean values of corneal power when comparing Cassini with Lenstar, but reported a significant difference in corneal power between Cassini and a Placido-based topographer. ${ }^{1}$ However, Klijn et al reported differences in corneal power between Cassini and Lenstar and Cassini and a Placido-based topographer, although the authors considered the differences to be of negligible clinical relevance. ${ }^{7}$ In addition, a comparison between Cassini and a Placido-based corneal topographer showed no differences in corneal power, ${ }^{2}$ although another study comparing Cassini and a Placido-based corneal topographer showed differences 
Axis
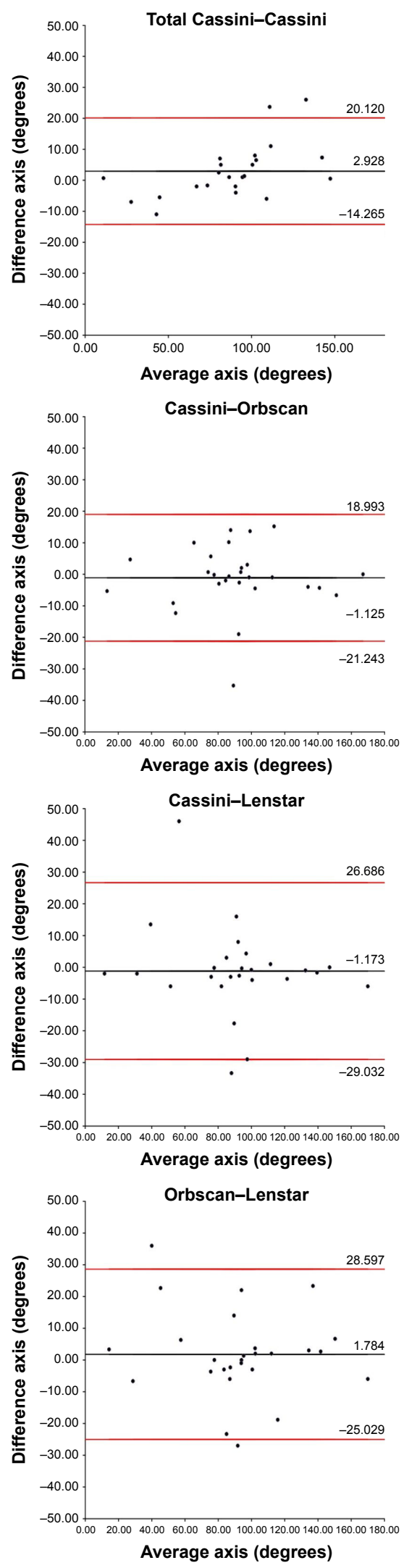

Astigmatism magnitude
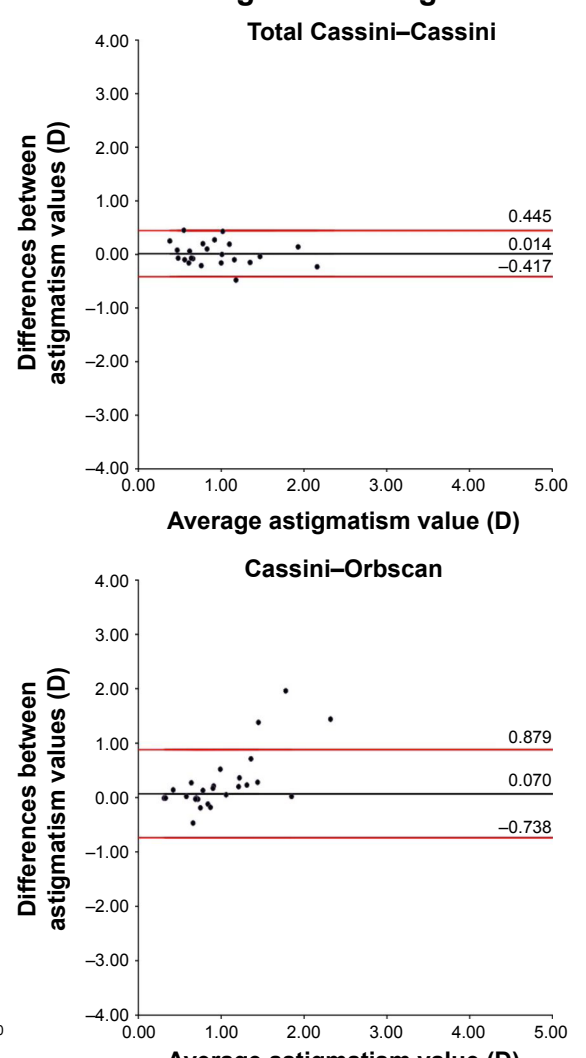

Average astigmatism value (D)

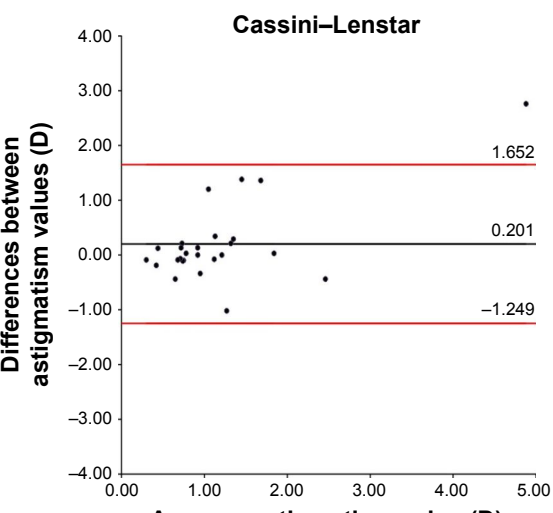

Average astigmatism value (D)

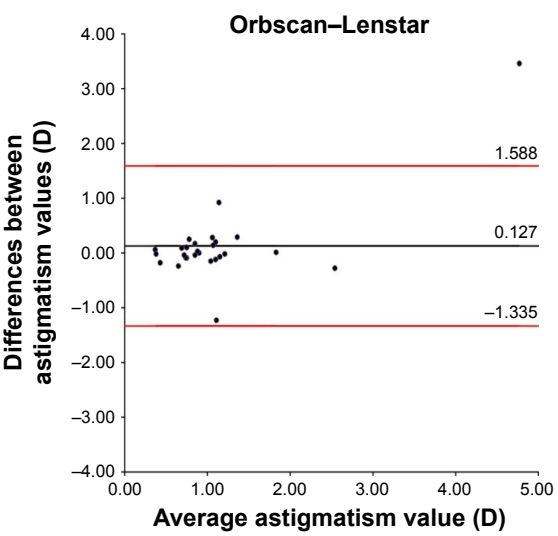

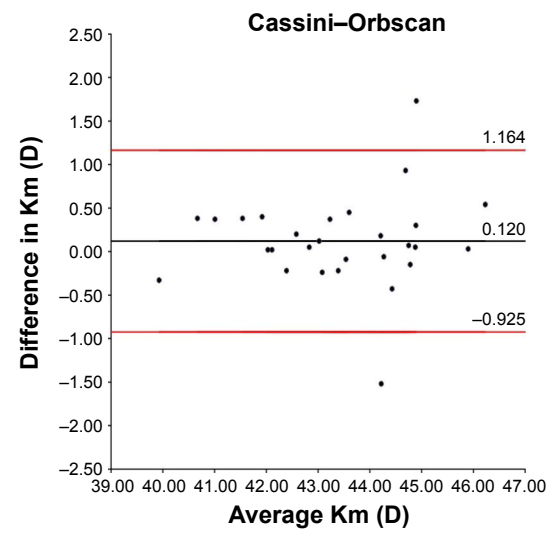
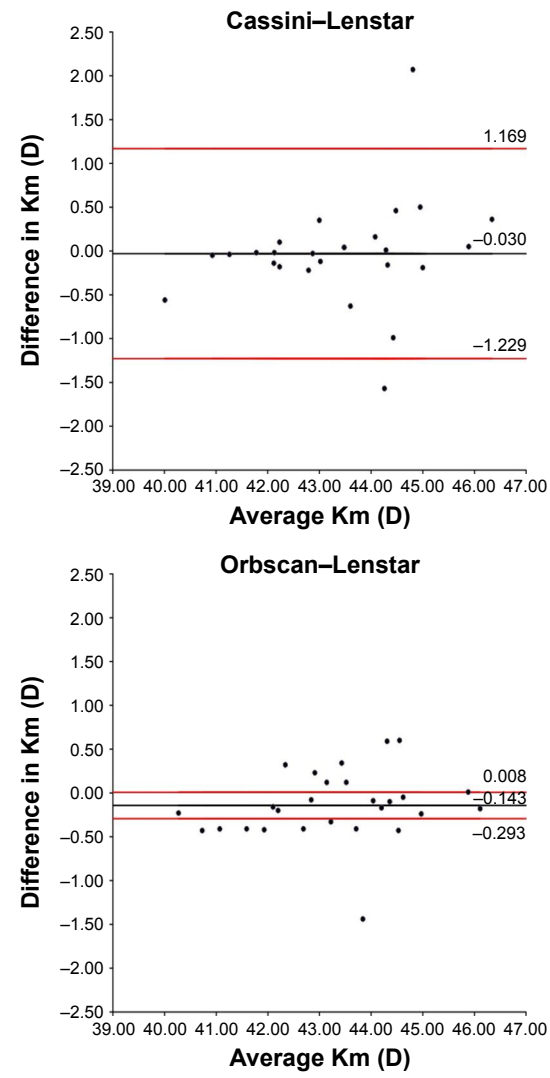

Figure I Bland-Altman plots for astigmatism axis, astigmatism magnitude, and $\mathrm{Km}$. The limits of agreement are shown by the red line. For astigmatism axis, the best agreement was between Total Cassini and Lenstar LS $900^{\circledR}$ and the worst was between Total Cassini and Cassini ${ }^{\circledR}$. For astigmatism magnitude, the best agreement was between Total Cassini and Cassini, and the worst was between Cassini and Lenstar. Regarding Km, the best agreement was between Cassini and Lenstar, and the worst was between Orbscan IIz ${ }^{\circledR}$ and Lenstar.

Abbreviation: $\mathrm{Km}$, mean keratometry. 
Table 2 Repeatability and comparability

\begin{tabular}{|c|c|c|c|c|c|c|}
\hline \multirow[t]{2}{*}{ Parameter } & \multicolumn{3}{|c|}{ Intra-keratometer (ICC, 95\% CI) } & \multicolumn{3}{|c|}{ Inter-keratometer (ICC, 95\% Cl) } \\
\hline & Cassini $^{\circledR}$ & Orbscan IIz ${ }^{\circledR}$ & Lenstar LS 900 ${ }^{\circledR}$ & Cassini-Orbscan & Cassini-Lenstar & Orbscan-Lenstar \\
\hline $\mathrm{KI}$ & $0.954(0.893-0.980)$ & $0.982(0.959-0.992)$ & $0.997(0.993-0.999)$ & $0.979(0.954-0.990)$ & $0.947(0.882-0.976)$ & $0.969(0.932-0.986)$ \\
\hline K2 & $0.814(0.609-0.918)$ & $0.979(0.953-0.991)$ & $0.997(0.993-0.999)$ & $0.954(0.90 I-0.979)$ & $0.949(0.886-0.977)$ & $0.968(0.930-0.985)$ \\
\hline Astigmatism & $0.913(0.806-0.963)$ & $0.955(0.90 \mathrm{I}-0.980)$ & $0.942(0.859-0.977)$ & $0.964(0.922-0.983)$ & $0.798(0.550-0.910)$ & $0.810(0.583-0.913)$ \\
\hline Axis & $0.984(0.962-0.993)$ & $0.970(0.933-0.987)$ & $0.978(0.945-0.991)$ & $0.976(0.948-0.989)$ & $0.960(0.911-0.982)$ & $0.964(0.92 I-0.984)$ \\
\hline j0 & $0.918(0.825-0.963)$ & $0.984(0.963-0.993)$ & $0.984(0.960-0.994)$ & $0.989(0.976-0.995)$ & $0.89 \mathrm{I}(0.76 \mathrm{I}-0.950)$ & $0.893(0.766-0.95 I)$ \\
\hline J45 & $0.621(0.313-0.812)$ & $0.920(0.827-0.965)$ & $0.950(0.877-0.980)$ & $0.522(-0.018$ to 0.776$)$ & $0.815(0.593-0.915)$ & $0.690(0.320-0.859)$ \\
\hline
\end{tabular}

Notes: All values are presented as median (minimum-maximum). All ICCs, both intra-keratometer and inter-keratometer, have $P<0.00 \mathrm{I}$. Repeatability was high for all measurement techniques and assessed parameters, with ICC $>0.900$, except for K2 and 445 measured by Cassini (ICC <0.900). Comparability was also high for most parameters (ICC >0.900), but several inter-keratometer comparisons showed ICC $<0.900$.

Abbreviations: ICC, intraclass correlation coefficient; $\mathrm{KI}$, minimum keratometry; $\mathrm{K} 2$, maximum keratometry.

in both $\mathrm{K} 1$ and $\mathrm{K} 2 .{ }^{8}$ We found no differences in the median values of $\mathrm{K} 1$ and $\mathrm{K} 2$ between Cassini and Orbscan or Lenstar, although the median values of $\mathrm{K} 1$ and $\mathrm{K} 2$ between Lenstar and Orbscan were different. Comparability was high between all assessment methods, with ICC $>0.900$.

Agreement analysis showed that the Cassini vs Lenstar agreement regarding $\mathrm{Km}$ showed the closest to 0 mean difference, but it also showed the highest range of LoA (2.4), which is clinically relevant, while Orbscan vs Lenstar agreement showed the highest mean difference and the lowest range of LoA. Similar or higher ranges of LoA have been reported for Cassini-Placido and Cassini-Lenstar for corneal power, leading the authors to discourage the interchangeable use of these devices. ${ }^{1,2,8}$

We also evaluated the median values of astigmatism magnitude and axis between Cassini and Orbscan or Lenstar, and they showed no differences. Comparability was also high regarding astigmatism magnitude and astigmatism axis for all paired devices, except for astigmatism magnitude measured by Cassini compared to Lenstar (ICC $=0.798$ ) and Lenstar compared to Orbscan (ICC $=0.810$ ). Once again, the results reported in the literature are different. Ventura et al found no differences in mean values of astigmatism magnitude when comparing Cassini with Lenstar, ${ }^{1}$ but in contrast with two studies comparing Cassini and two different Placidobased corneal topographers, both showing differences in astigmatism magnitude. ${ }^{2,8}$ Our results are further supported by agreement analysis, which showed that, for astigmatism magnitude, the agreement between Cassini and Lenstar showed the highest mean difference, with the agreement between the Orbscan and Lenstar showing the highest range of LoA. Similar or higher ranges of LoA have been reported for Cassini-Placido and Cassini-Lenstar for astigmatism magnitudes. ${ }^{1,2,8}$ Regarding astigmatism axis, comparisons showed a wide data spread.

As for $\mathrm{J} 0$ and J45, our results showed differences in J45 between Cassini and Lenstar, with Lenstar showing higher values. There were also differences in the median values of J0 and J45 between Total Cassini and Cassini. However, Ventura et al found no differences in the mean values of J45 when comparing Cassini with Lenstar. ${ }^{1}$ As for comparability, it was only high for J0 between Cassini and Orbscan (ICC $=0.989$ ), with all other comparisons showing ICC $<0.900$. Although all comparisons showed a $P<0.001$, J45 between Cassini and Orbscan and between Lenstar and Orbscan were notably low (ICC $=0.522$ and ICC $=0.690$, respectively).

These different results may be accounted for due to the different population samples, different operators, and different Placido-based devices. Given there is no gold standard device for these measurements, no conclusions can be drawn regarding which device is the most accurate. Agreement analysis showed a wide data spread, suggesting that these devices should not be used interchangeably, despite the high ICC values.

\section{Repeatability}

Repeatability was high for all measurement techniques and assessed parameters, with ICC $>0.900$, except for K2 and J45 measured by Cassini. Two studies assessing Cassini repeatability concluded that Cassini has enhanced precision, further improving astigmatism magnitude, astigmatism axis repeatability, ${ }^{11}$ and keratometry, ${ }^{10}$ even in LASIK-treated, keratoconic, and cross-linked corneas. ${ }^{10}$ However, other studies have shown different results. One study concluded that, although Cassini provided highly repeatable measurements, it had a worse repeatability than Lenstar in all parameters, with Lenstar showing the best repeatability of all studied devices. ${ }^{1}$ However, another study reported a relatively low repeatability of corneal power measurements with Cassini, but a higher repeatability of cylinder measurements compared to both Lenstar and a Placido-based topographer. ${ }^{7}$ In contrast, Hidalgo et al showed a good repeatability for Cassini and Placido, both for keratometry and astigmatism. ${ }^{8}$ 
A comparison between Lenstar and two Placido-based topographers showed that Lenstar and one of the Placidobased topographers showed a reasonable repeatability for corneal power, J0, and J45, while the other Placido-based topographer showed poor repeatability for J45. ${ }^{3}$ This same study showed that one of the corneal Placido-based topographers had statistically higher repeatability for corneal astigmatism and Lenstar had lower repeatability, ${ }^{3}$ but another study concluded that Lenstar showed acceptable repeatability. ${ }^{5}$ Other authors have reported a very high repeatability for Placido-based topographers, with ICC $>0.990$ for $\mathrm{K} 1, \mathrm{~K} 2,{ }^{4,6}$ and $\mathrm{Km} .{ }^{4}$ Analysis of our results shows that the best repeatability for $\mathrm{K} 1$ and $\mathrm{K} 2$ was achieved by Lenstar, and the worst for K2 and J45 measured by Cassini. All other 95\% CIs show overlap.

In conclusion, the best repeatability for $\mathrm{K} 1$ and $\mathrm{K} 2$ was achieved by Lenstar. All measurement techniques also showed a high comparability regarding $\mathrm{K} 1$, K2, and astigmatism axis. However, the wide data spread suggests that these devices should not be used interchangeably. Further research is required to assess the validity and precision of these measurements obtained with different devices.

\section{Acknowledgment}

The authors thank Ophtec Portugal for the funding provided for this paper, which was used to access all necessary scientific bibliography.

\section{Disclosure}

The authors report no conflicts of interest in this work.

\section{References}

1. Ventura BV, Al-Mohtaseb Z, Wang L, et al. Repeatability and comparability of corneal power and corneal astigmatism obtained from a point-source color light-emitting diode topographer, a Placido-based corneal topographer, and a low-coherence reflectometer. $J$ Cataract Refract Surg. 2015;41(10):2242-2250.
2. Ventura BV, Wang L, Ali SF, et al. Comparison of corneal power, astigmatism, and wavefront aberration measurements obtained by a point-source color light-emitting diode-based topographer, a Placidodisk topographer, and a combined Placido and dual Scheimpflug device. J Cataract Refract Surg. 2015;41(8):1658-1671.

3. Fityo S, Bühren J, Shajari M, et al. Keratometry versus total corneal refractive power: analysis of measurement repeatability with 5 different devices in normal eyes with low astigmatism. J Cataract Refract Surg. 2016;42(4):569-576.

4. Hua Y, Xu Z, Qiu W, et al. Precision (repeatability and reproducibility) and agreement of corneal power measurements obtained by Topcon KR-1W and iTrace. PLoS One. 2016;11(1):e0147086.

5. Visser N, Berendschot TT, Verbakel F, et al. Comparability and repeatability of corneal astigmatism measurements using different measurement technologies. J Cataract Refract Surg. 2012;38(10): 1764-1770.

6. Wang Q, Savini G, Hoffer KJ, et al. A comprehensive assessment of the precision and agreement of anterior corneal power measurements obtained using 8 different devices. PLoS One. 2012;7(9):e45607.

7. Klijn S, Reus NJ, Sicam VA. Evaluation of keratometry with a novel Color-LED corneal topographer. J Refract Surg. 2015;31(4):249-256.

8. Hidalgo IR, Rozema JJ, Dhubhghaill SN, et al. Repeatability and interdevice agreement for three different methods of keratometry: Placido, Scheimpflug, and color LED corneal topography. J Refract Surg. 2015; 31(3):176-181.

9. Ferreira TB, Ribeiro FJ. A novel color-LED corneal topographer to assess astigmatism in pseudophakic eyes. Clin Ophthalmol. 2016;10: $1521-1529$.

10. Kanellopoulos AJ, Asimellis G. Color light-emitting diode reflection topography: validation of keratometric repeatability in a large sample of wide cylindrical-range corneas. Clin Ophthalmol. 2015;9:245-252.

11. Kanellopoulos AJ, Asimellis G. Distribution and repeatability of corneal astigmatism measurements (magnitude and axis) evaluated with color light emitting diode reflection topography. Cornea. 2015;34(8): 937-944.

12. Lenstar LS $900^{\circledR}$ Biometer Instruction Manual, Section 4.3.2. Koeniz: Haag-Streit AG.

13. Kanellopoulos AJ, Asimellis G. Forme fruste keratoconus imaging and validation via novel multi-spot reflection topography. Case Rep Ophthalmol. 2013;4(3):199-209.

14. Thibos LN, Horner D. Power vector analysis of the optical outcome of refractive surgery. $J$ Cataract Refract Surg. 2001;27(1):80-85.

15. Freitas Gde O, Ambrosio R Jr, Ramos I, et al. Astigmatic vector analysis of posterior corneal surface: a comparison among healthy, forme fruste, and overt keratoconic corneas. Am J Ophthalmol. 2016;167:65-71.

16. McAlinden C, Khadka J, Pesudovs K. Statistical methods for conducting agreement (comparison of clinical tests) and precision (repeatability or reproducibility) studies in optometry and ophthalmology. Ophthalmic Physiol Opt. 2011;31(4):330-338.
Clinical Ophthalmology

\section{Publish your work in this journal}

Clinical Ophthalmology is an international, peer-reviewed journal covering all subspecialties within ophthalmology. Key topics include: Optometry; Visual science; Pharmacology and drug therapy in eye diseases; Basic Sciences; Primary and Secondary eye care; Patient Safety and Quality of Care Improvements. This journal is indexed on Submit your manuscript here: http://www.dovepress.com/clinical-ophthalmology-journal

\section{Dovepress}

PubMed Central and CAS, and is the official journal of The Society of Clinical Ophthalmology (SCO). The manuscript management system is completely online and includes a very quick and fair peer-review system, which is all easy to use. Visit http://www.dovepress.com/ testimonials.php to read real quotes from published authors. 